\title{
Article \\ Throughput and Packet Loss Probability Analysis of Long Range Wide Area Network
}

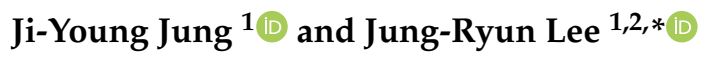 \\ 1 School of Electrical and Electronics Engineering, Chung-Ang University, 84 Heukseok-ro, Dongjak-gu, \\ Seoul 06974, Korea; jiyoung@cau.ac.kr \\ 2 Department of Intelligent Energy and Industry, Chung-Ang University, 84 Heukseok-ro, Dongjak-gu, \\ Seoul 06974, Korea \\ * Correspondence: jrlee@cau.ac.kr; Tel.: +82-2-820-5820
}

Citation: Jung, J.-Y.; Lee, J.-R.

Throughput and Packet Loss

Probability Analysis of Long Range

Wide Area Network. Appl. Sci. 2021,

11, 8091. https://doi.org/10.3390/

app11178091

Academic Editors: Javier Pereira and Manuel Francisco González Penedo

Received: 7 July 2021

Accepted: 25 August 2021

Published: 31 August 2021

Publisher's Note: MDPI stays neutral with regard to jurisdictional claims in published maps and institutional affiliations.

Copyright: (C) 2021 by the authors. Licensee MDPI, Basel, Switzerland. This article is an open access article distributed under the terms and conditions of the Creative Commons Attribution (CC BY) license (https:// creativecommons.org/licenses/by/ $4.0 /)$.

\begin{abstract}
Long Range Wide Area Network (LoRaWAN) is the one of the promising low power wide area network (LPWAN) technologies at present and is expected to grow in the foreseeable future as a tool to provide connectivity among small things. In this paper, we present a simple analytical model to compute the throughput and packet loss probability of Medium Access Control (MAC) for Class-A of LoRaWAN. This analysis results can be used as a reference for deploying the appropriate number of end-devices (EDs) that can be accepted in a gateway (GW) while maximizing network throughput or guaranteeing the packet loss rate of EDs.
\end{abstract}

Keywords: Internet of Things; low power wide area network; long range wide area network; Markov chain model

\section{Introduction}

Since the emergence of the Internet of Things concept, several communication technologies were proposed to provide various IoT services. LoRaWAN is the one of the LPWA network technologies at present and is considered as the basis of IoT application in many industries [1]. LoRaWAN provides three different classes for EDs to address the various needs of applications: Class-A, Class-B, and Class-C. Class-A is a default mode which should be implemented in all EDs. When an ED has an uplink packet to transmit, it immediately selects a frequency channel randomly and transmits the packet. Uplink transmission is followed by two short downlink receive windows: window RX1 after the receive delay 1 and window $R X 2$ after the receive delay 2 , respectively. When a GW has a downlink packet to transmit, it transmits the packet only through these two windows. The GW transmits the downlink packet preferentially through window RX1 over the same channel as the uplink channel. If the GW fails to transmit through RX1, it can re-transmit the packet through window RX2 over another downlink channel dedicated for the downlink receive window. It is noticed that an ED of Class-A sleeps at all times except uplink and two short downlink receive windows, which provides an ED with the highest energy efficiency among all classes of LoRaWAN.

In LoRaWAN, unlike cellular networks, it is not easy to realize the elaborate access control and QoS support for EDs because of the limited available bandwidth and processing capability. Moreover, as the network size is getting larger, the control of large-scale network becomes difficult. In order to efficiently control the transmission collision of uplink packet originating from numerous uncontrollable EDs in LoRaWAN, it is essential to evaluate the performance of the uplink packet transmission taking into account the characteristics of Class-A of LoRaWAN.

Many research studies on LoRaWAN have been conducted until now. Some studies measured the performance of LoRaWAN ED considering the distance between an ED and a GW or evaluating performance of LoRaWAN system as a function of the number of 
EDs [2-6]. In addition, various efforts have been made to improve the network system capacity. In Reference [7], considering that the operation of Class-A in LoRaWAN is based on pure-Aloha approach, the author proposed the algorithm to improve the system capacity by employing slotted-Aloha approach in the standard. Analytical models for performance metrics for uplink packet transmission were provided in Reference $[8,9]$. To overcome the performance degradation of pure Aloha in LoRaWAN as the network size grows, listen before talk medium access strategy was proposed in Reference [10]. In this paper, we analyze the performance of uplink packet transmission of LoRaWAN Class-A mode using Markov model and evaluate the uplink packet transmission performance in terms of throughput and packet loss probability.

\section{LoRaWAN System Model}

A typical LoRa physical layer provides configuration parameters, including carrier frequency, spreading factor, bandwidth, and coding rate. Actual packet transmission time in LoRaWAN is determined by spreading factor, bandwidth, and coding rate.

The carrier frequency (CF) is the center frequency that can be programmed in units of $61 \mathrm{~Hz}$ between $137 \mathrm{MHz}$ and $1024 \mathrm{MHZ}$. Spreading Factor (SF) is a ratio between a symbol rate and a chip rate, and has a value ranging from 6 to 12. The number of chips per symbol is defined as $2^{S F}$. As SF increases, the transmission radius of an ED increases due to the increase of SNR. However, in this case, the packet transmission time also increases; therefore, the energy consumption of the ED increases as a consequence. Bandwidth (BW) is the frequency width of the transmission band and the available bandwidth is $500 \mathrm{kHz}, 250 \mathrm{kHz}$, and $125 \mathrm{kHz}$ in LoRaWAN. As BW increases, the packet transmission rate increases, but the SNR decreases due to an additional noise. LoRaWAN includes a forward error correction (FEC) code that is used for controlling errors in data transmission. Coding rate (CR) of the FEC is the proportion of the useful information and the total data bits and it can be set to either 4/5,4/6,4/7, and $4 / 8$ in LoRaWAN. As CR increases, the information protection function improves, but the transmission time increases due to large amount of redundant bits.

In LoRaWAN, the packet transmission time is denoted by a time-on-air (ToA), which is the sum of the length of the preamble $\left(T_{p b}\right)$ and the length of the actual packet payload $\left(T_{p l}\right)$ [11], which is given by

$$
T o A=T_{p b}+T_{p l} .
$$

Let $n_{p b}, n_{p l}, T_{S}, S F$, and $C R$ be the number of preamble symbols for LoRaWAN, the number of payload bits, the duration of a symbol, the spreading factor, and the coding rate, respectively. Then $T_{p b}$ and $T_{p l}$ are calculated by

$$
T_{p b}=\left(n_{p b}+4.25\right) \cdot T_{S}, \quad T_{p l}=n_{p l} \cdot T_{S},
$$

where $n_{p l}=8+\max \left(\left\lceil\frac{8 P L-4 S F+28+16 C R C-20 I H}{4(S F-2 D E)}\right\rceil(C R+4), 0\right), T_{S}=\frac{1}{R_{S}}$, and $\lceil\cdot\rceil$ is a ceiling function. In (2), CRC = 1 if cyclic redundancy check functionality is enabled, or CRC $=0$ otherwise. IH specifies the presence of PHY header; $\mathrm{IH}=1$ (0) for implicit (explicit) operation mode. DE indicates the using of data rate optimization; $\mathrm{DE}=1$ if enabled, or $\mathrm{DE}=0$ otherwise. $R_{S}$ is the symbol rate composed of the $\mathrm{BW}$ and the number of chips per symbol, which is calculated by

$$
R_{S}=\frac{B W}{2^{S F}} .
$$

PL is the number of bytes in the payload, and is calculated as

$$
\begin{aligned}
P L= & M H+F H_{\text {addr }}+F H_{c t l}+F H_{c n t} \\
& +F H_{o p t}+F_{p o r t}+F R M_{p l}+M I C,
\end{aligned}
$$

where $M H, F H_{a d d r}, F H_{c t l}, F H_{c n t}, F H_{o p t}, F_{p o r t}, F R M_{p l}$, and $M I C$ are the MAC header length, the frame header address field length, the frame control field length, the frame counter 
field length, the optional field's length, the port identifier length, the frame payload length, and the message integrity code length, respectively [11]. Here, all parameters except $F R M_{p l}$ have fixed value; therefore, (4) is re-written by

$$
P L=13+F R M_{p l} .
$$

By combining (1), (2), (3), and (5), we have

$$
\text { To } A=\frac{2^{S F}}{B W}\left(20.25+\max \left(\left\lceil\frac{8\left(13+F R M_{p l}\right)-4 S F+44}{4(S F-2 D E)}\right\rceil \cdot 5,0\right)\right),
$$

where $D E=0$ for $6 \leq S F \leq 10$, and $D E=1$ for $11 \leq S F \leq 12$. In LoRaWAN, $S F$ is determined by signal to noise ratio (SNR) given by

$$
S N R_{d B}=10 \log _{10}\left(\frac{P_{\text {signal }}}{P_{\text {noise }}}\right)=P_{\text {signal }, d B}-P_{\text {noise }, d B} .
$$

Here, $P_{\text {signal }, d B}$ is calculated by

$$
P_{\text {signal }, d B}=P_{T X}+G_{T X}-L_{T X}-L_{P L}-L_{M}+G_{R X}-L_{R X},
$$

where $P_{T X}, G_{T X}, L_{T X}, L_{P L}, L_{M}, G_{R X}$, and $L_{R X}$ are tx output power, tx antenna gain, transmitter losses (coax, connectors), path loss, miscellaneous losses (fading margin, body loss, polarization mismatch), rx antenna gain, and receiver losses, respectively.

We simplify (8) by combining all general gains and losses as GL, which results in

$$
P_{\text {signal }, d B}=P_{T X}-L_{P L}+G L .
$$

LoRaWAN uses a log-distance path loss model, which is modeled for inside a building or densely populated areas. In a log-distance path loss model, the path loss, $L_{P L}$, is defined [12] as

$$
L_{P L}=L_{P L\left(d_{0}\right)}+10 \gamma \log \left(d / d_{0}\right)+X_{\sigma}
$$

where $L_{P L\left(d_{0}\right)}$ is the path loss at the reference distance $d_{0}, \gamma$ is the path loss exponent, and $X_{\sigma}$ is a normal random variable with zero mean, reflecting the attenuation caused by flat fading, and $d$ is the distance between the ED and the GW, respectively. The noise power $P_{n o i s e, d B}$ is defined as a function of the bandwidth (BW) and is given by [13]

$$
P_{\text {noise }, \mathrm{dB}}=-174+10 \log _{10}(B W) .
$$

$P_{T X}$ and $G L$ are set to $14 \mathrm{dBm}$ and 0 , respectively, by referring to the LoRa module specification. We use $L_{P L\left(d_{0}\right)}=127.41 \mathrm{~dB}, d_{0}=1 \mathrm{~km}$, and $\gamma=2.08$ as in Reference [12]. Table 1 shows the measured distance between a GW and an ED obtained by combining (7), (9), (10), and (11), and this table is used to determine the SF of an ED for uplink transmission. 
Table 1. Measured distance between a GW and an ED for different SF, SNR, and BW.

\begin{tabular}{ccccc}
\hline \multirow{2}{*}{ SF } & SNR & \multicolumn{3}{c}{ BW } \\
\cline { 3 - 5 } & & $\mathbf{1 2 5} \mathbf{~} \mathbf{H z}$ & $\mathbf{2 5 0} \mathbf{~ k H z}$ & $\mathbf{5 0 0} \mathbf{~ k H z}$ \\
\hline 6 & $-5 \mathrm{~dB}$ & $5.045 \mathrm{~km}$ & $3.615 \mathrm{~km}$ & $2.591 \mathrm{~km}$ \\
\hline 7 & $-7.5 \mathrm{~dB}$ & $6.654 \mathrm{~km}$ & $4.768 \mathrm{~km}$ & $3.417 \mathrm{~km}$ \\
\hline 8 & $-10 \mathrm{~dB}$ & $8.775 \mathrm{~km}$ & $6.288 \mathrm{~km}$ & $4.506 \mathrm{~km}$ \\
\hline 9 & $-12.5 \mathrm{~dB}$ & $11.574 \mathrm{~km}$ & $8.293 \mathrm{~km}$ & $5.943 \mathrm{~km}$ \\
\hline 10 & $-15 \mathrm{~dB}$ & $15.263 \mathrm{~km}$ & $10.938 \mathrm{~km}$ & $7.838 \mathrm{~km}$ \\
\hline 11 & $-17.5 \mathrm{~dB}$ & $20.132 \mathrm{~km}$ & $14.426 \mathrm{~km}$ & $10.33 \mathrm{~km}$ \\
\hline 12 & $-20 \mathrm{~dB}$ & $26.550 \mathrm{~km}$ & $19.026 \mathrm{~km}$ & $13.634 \mathrm{~km}$ \\
\hline
\end{tabular}

\section{Markov Chain Model}

In LoRaWAN, time, frequency, and spreading factor are orthogonal factors to each other, so packet collision occurs only when two or more EDs transmit a packet at the same time using the same frequency channel and same spreading factor. Suppose that there are $N$ EDs associated with a gateway. We define $N_{i j}$ as the number of EDs who uses the spreading factor $S F_{i}(6 \leq i \leq 12)$, and the carrier frequency $C F_{j}(1 \leq j \leq 6)$. The state of the uplink channel is divided into three states: a success state during when an uplink packet is successfully transmitted, a collision state during when a collision happens, and an idle state in which there are no packet transmission activities. Let $b_{i, j}(t) \in[0, S]$ be the stochastic process representing the time-slot counter for the uplink packet transmission of an ED with $S F_{i}$ and $C F_{j}$ at slot time $t$, where $S$ is the number of time slots required to transmit an uplink packet. In this model, a discrete and integer-type time scale is adopted, and the time-slot counter used for the uplink packet transmission decreases at the beginning of each slot time (It is noticed that this discrete time-scale does not directly relates to the system time).

For given $i$ and $j$, we define $s_{i, j}(t)$ as the stochastic process representing the number of EDs with $S F_{i}$ and $C F_{j}$ which transmit or try to transmit a uplink packet at time $t$. Here, we construct the discrete-time bi-dimensional Markov chain $\left\{s_{i, j}(t), b_{i, j}(t)\right\}$ as depicted in Figure 1. It is noticed that this Markov chain describes only the success and collision states except idle state in order to simplify the Markov chain model. Hereafter, considering orthogonalities of spreading factor and carrier frequency, we simply denote the above Markov chain as $\{s(t), b(t)\}$, omitting the lower index for given $i$ and $j$.

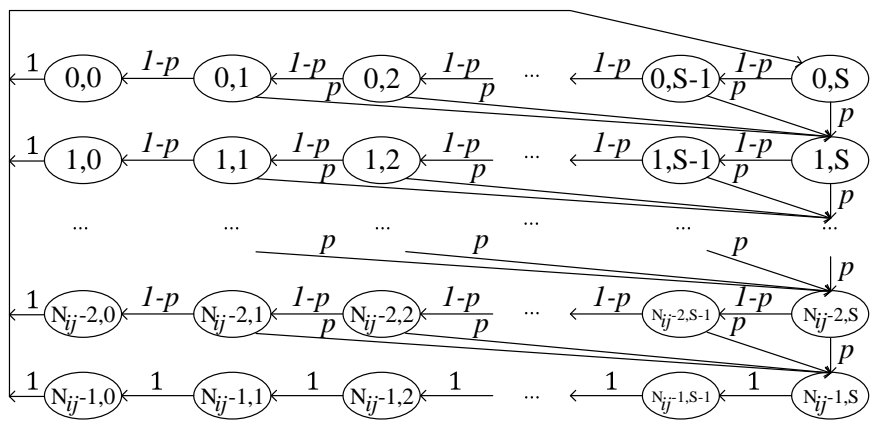

Figure 1. Markov chain model.

ITU-T describes that many IoT traffic models have a common characteristic of frequent short-packet transmission with a Poisson distribution, based on the assumption that the reporting from EDs are uncorrelated[14]. Therefore, we approximate the distributions of 
the length of uplink packet transmission interval using exponential distribution. Let $p$ be the probability of an ED to try to transmit uplink packet at a time slot. Then, $p$ is given by

$$
p=1-e^{-\lambda}, \text { where } \lambda=\frac{N_{i j}}{T_{U}},
$$

where $T_{U}$ is the length of uplink packet transmission interval of a node. In this Markov chain, the one-step transition probabilities are calculated by

$$
\begin{aligned}
& P\{l, k \mid l, k+1\}=1-p \text { for } 0 \leq k \leq S-1,0 \leq l \leq N_{i j}-1 \\
& P\{0, S \mid l, 0\}=1 \text { for } 0 \leq l \leq N_{i j}-1, \\
& P\{l, S \mid l-1, k\}=p \text { for } 1 \leq k \leq S, 1 \leq l \leq N_{i j}-1, \\
& P\left\{N_{i j}-1, k \mid N_{i j}-1, k+1\right\}=1 \text { for } 0 \leq k \leq S-1 .
\end{aligned}
$$

Let $b_{l, k}$ be the stationary distribution of $\{s(t), b(t)\}$, defined by $b_{l, k}=\lim _{t \rightarrow \infty}$ for $P\{s(t)=$ $l, b(t)=k\}$ for $0 \leq l \leq N_{i j}-1,0 \leq k \leq S$. Then, we can express $b_{l, k}$ as

$$
b_{l, k}=(1-p)^{S-k} b_{l, S} \text { for } 0 \leq l \leq N_{i j}-2 .
$$

In addition, $b_{N_{i j}-1, k}$ for $0 \leq k \leq S-1$ can be expressed by

$$
b_{N_{i j}-1, k}=b_{N_{i j}-1, S} \text {. }
$$

Notice that starting in state $b_{N_{i j}-1, S}$ will be in state $b_{N_{i j}-1, k}$ after $S$ steps because the length of uplink packet transmission interval is extremely larger than the length of $T o A$. From the chain regularity, we have

$$
b_{0, S}=\sum_{l=0}^{N_{i j}-1} b_{l, 0}, \quad b_{l, S}=\sum_{k=1}^{S} p b_{l-1, k}
$$

From (14)-(16), we have

$$
1=\sum_{l=0}^{N_{i j}-1} \sum_{k=0}^{S} b_{l, k}=\sum_{l=0}^{N_{i j}-2} \sum_{k=0}^{S-1}(1-p)^{S-k} q^{l} b_{0, S}+S q^{N_{i j}-1} b_{0, S}+b_{0, S}+\sum_{l=1}^{N_{i j}-1} q^{l} b_{0, S},
$$

where $q=\sum_{k=1}^{S} p(1-p)^{S-k}$. Thus, $b_{0,0}$ is expressed as a function of the probability of an ED to try to transmit uplink packet at a time slot $p$, which is given by

$$
b_{0,0}=\left[\sum_{l=0}^{N_{i j}-2} \sum_{k=0}^{S-1}(1-p)^{S-k} q^{l}+S q^{l}+1+\sum_{l=1}^{N_{i j}-1} q^{l}\right]^{-1} .
$$

$b_{0,0} \times S$ is the probability of the uplink channel state going into success state under the condition that there are only the success and collision states except idle state.

Let $P_{T}$ and $P_{S}$ be the probability of an ED being in an uplink packet transmission and the probability of an ED being in sleep and RX windows, respectively. Then, $P_{T}$ and $P_{S}$ are expressed by

$$
P_{T}=\frac{T o A}{T_{U}}, \quad P_{S}=1-\frac{T o A}{T_{U}} .
$$

Let $P_{\text {Idle }}\left(S F_{i}, C F_{j}\right)$ and $P_{\text {Busy }}\left(S F_{i}, C F_{j}\right)$ be the probabilities of the $j$-th carrier frequency channel being in idle and busy states in view of EDs with given $S F_{i}$, respectively. Then, we have

$$
P_{\text {Idle }}\left(S F_{i}, C F_{j}\right)=P_{S}^{N_{i j}}=1-P_{\text {Busy }}\left(S F_{i}, C F_{j}\right) .
$$


We call $T\left(S F_{i}, C F_{j}\right)$ as the normalized throughput of the channel using the $j$-th carrier frequency and spreading factor $i$, which is defined as the fraction of time during when the channel is used to transmit packets without collisions. Then, it is calculated by

$$
T\left(S F_{i}, C F_{j}\right)=P_{\text {Busy }} \cdot b_{0,0} \cdot S .
$$

The packet loss probability $\operatorname{PLP}\left(S F_{i}, C F_{j}\right)$ is defined as the percentage of packets lost with respect to packets sent, and then it is calculated by

$$
\operatorname{PLP}\left(S F_{i}, C F_{j}\right)=1-\frac{b_{0,0}}{\sum_{l=0}^{N_{i j}-1} b_{l, S}} .
$$

\section{Performance Evaluation}

For performance evaluation, we employ the EU (863-870MHz) standard which has been mostly studied. In Table 2, various transmission options from 1 to 7 used in the EU standard are shown including spreading factor, bandwidth, coding rate, and frame payload size. Combining these parameter sets and (1) results in ToA values, which are calculated in the rightmost column in this table. It is noted that frame payload size is set to the maximum size available defined in the EU standard. We set $T_{U}$ as $30 \mathrm{~s}$, and the length of the receive delay 1 as 1 second. We assume $N$ EDs associated with a GW. The geographical position of each ED is randomly determined within the maximum transmission radius of a GW. $\mathrm{SF}$ of an ED is determined according to SINR of the ED based on the distance between the ED and the fading channel gain. $A_{i}, i \in(1,7)$ denotes the set with nodes using the transmission option $i$ in Table 2. $A_{A l l}$ denotes the set of all nodes in a network and is expressed as $\cup_{i} A_{i}$.

Table 2. Transmission options in EU(863-870MHz) standard.

\begin{tabular}{cccccc}
\hline No & SF & BW (kHz) & CR & FRM $_{\boldsymbol{p l}}$ (Bytes) & ToA (Sec) \\
\hline 1 & 7 & 250 & $4 / 5$ & 242 & 0.199 \\
2 & 7 & 125 & $4 / 5$ & 242 & 0.399 \\
3 & 8 & 125 & $4 / 5$ & 242 & 0.707 \\
4 & 9 & 125 & $4 / 5$ & 115 & 0.676 \\
5 & 10 & 125 & $4 / 5$ & 51 & 0.698 \\
6 & 11 & 125 & $4 / 5$ & 51 & 1.560 \\
7 & 12 & 125 & $4 / 5$ & 51 & 2.793 \\
\hline
\end{tabular}

Figure 2 shows the normalized throughput for $A_{i}$ and $A_{A l l}$. Here, the normalized throughput for $A_{x}$ is the average throughput of EDs in $A_{x}$ which is obtained by the sum of the throughput of all EDs in $A_{x}$ divided by the cardinality of $A_{x}$. The result shows that the analysis result coincides with the simulation result. As the number of EDs in a GW increases, traffic load increases accordingly, which results in the increase of the normalized throughput at the initial phase for each region $A_{i}$. However, the normalized throughput decreases gradually after it reaches its peak performance because of increased packet collisions and packet retransmissions. We can see that the peak value of the throughput is reached quickly as the length of ToA increases. 


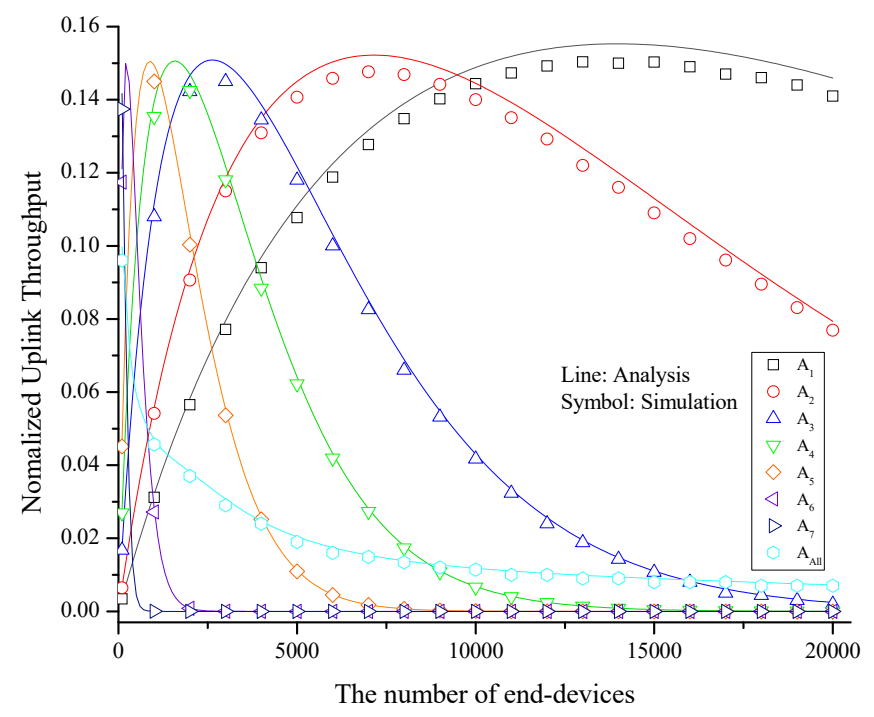

Figure 2. Normalized throughput as a function of the number of EDs.

Figure 3 shows the packet loss probability of $A_{i}$ and $A_{A l l}$ as a function of $N$. As the number of EDs in a GW increases, packet loss probability increases steadily because of increased packet collisions and packet retransmissions. We can see that the packet loss probability increases more rapidly as the length of ToA increases, and the overall packet loss probability $A_{A l l}$ increases as the number of EDs in a GW increases.

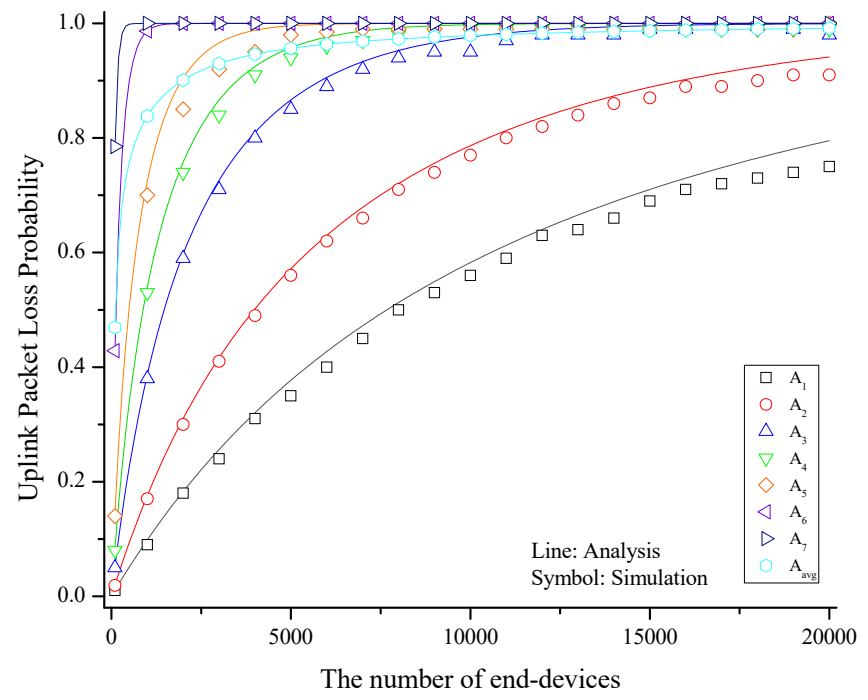

Figure 3. Packet Loss Probability as a function of the number of EDs.

\section{Conclusions}

In this paper, we studied the performance evaluation of uplink transmission in LoRaWAN Class-A mode. We provided an analytical evaluation of the normalized throughput and packet loss probability of LoRaWAN MAC using Markov chain model, and we evaluated the performances of the normalized throughput and the packet loss probability as the number of EDs in a GW. The results showed a reference for deploying the appropriate number of EDs that can be accepted in a GW while maximizing network throughput or guaranteeing the packet loss rate of EDs. For example, suppose that a network mainly consists of $A_{2}$ nodes. In this case, the number of nodes achieving the maximum throughput is around 7000, while satisfying the packet loss probability of around 0.65 . If the network operator wishes to restrict the packet loss rate within 0.3, the number of EDs should be less than 2000 with 
the normalized throughput of 0.09 . We expect this study to contribute to understanding LoRaWAN MAC and providing guidance on system performance analysis of LoRaWAN.

Author Contributions: J.-Y.J. contributed the paper by deriving results of simulation and by making the draft version of the paper. J.-R.L. was responsible for the main idea, theoretical analysis, coordination, revision, and proof reading of the paper. Both authors have read and agreed to the published version of the manuscript.

Funding: This work was supported by the MSIT (Ministry of Science and ICT), Korea, under the ITRC support program (IITP-2021-2018-0-01799) supervised by the IITP (Institute for Information and communications Technology Planning and Evaluation, in part by the Korea Institute of Energy Technology Evaluation and Planning (KETEP) and the Ministry of Trade, Industry and Energy (MOTIE) of the Republic of Korea under Grant 20214000000280, and in part by the National Research Foundation of Korea (NRF) grant funded by the Korea government (MEST) under Grant NRF2020R1A2C1010929.

Data Availability Statement: Not applicable.

Conflicts of Interest: The authors declare no conflict of interest.

\section{References}

1. Raza, U.; Kulkarni, P.; Sooriyabandara, M. Low power wide area networks: An overview. IEEE Commun. Surv. Tutor. 2017, 19, 855-873. [CrossRef]

2. Mohamed Aref and Axel Sikora, Free Space Range Measurements with Semtech LoRaTM Technology. In Proceedings of the 2014 IEEE IDAACS-SWS, Offenburg, Germany, 11-12 September 2014; pp. 19-23.

3. Petajajarvi, J.; Mikhaylov, K.; Roivainen, A.; Hanninen, T. On the Coverage of LPWANs: Range Evaluation and Channel Attenuation Model for LoRa techonology. In Proceedings of the 2015 ITST, Copenhagen, Denmark, 2-4 December 2015; pp. 55-59.

4. Petajajarvi, J.; Mikhaylov, K.; Hamalainen, M.; Iinatti, J. Evaluation of LoRa LPWAN Technology for Remote Health and Wellbeing Monitoring. In Proceedings of the 2016 ISMICT, Worcester, MA, USA, 20-22 March 2016; pp. 1-5.

5. Neumann, P.; Montavont, J.; Noel, T. Indoor deployment of low-power wide area networks (LPWAN): A LoRaWAN case study. In Proceedings of the 2016 IEEE WiMob, NewYork, NY, USA, 17-19 October 2016; pp. 1-8.

6. Wendt, T.; Volk, F.; Mackensen, E. A benchmark survey of long range (LoTaTM) spread spectrum communication at $2.45 \mathrm{GHz}$ for safety applications. In Proceedings of the 2015 WAMICON, Melbourne, FL, USA, 13-15 April 2015; pp. 1-4.

7. Polonelli, T.; Brunelli, D.; Marzocchi, A.; Benini, L. Slotted ALOHA on LoRaWAN-Design, Analysis, and Deployment. Sensors 2019, 19, 838. [CrossRef] [PubMed]

8. Sørensen, R.B.; Kim, D.M.; Nielsen, J.J.; Popovski, P. Analysis of Latency and MAC-Layer Performance for Class A LoRaWAN. IEEE Wirel. Commun. Lett. 2017, 6, 566-569. [CrossRef]

9. Khan, F.H.; Jurdak, R.; Portmann, M. A Model for Reliable Uplink Transmissions in LoRaWAN. In Proceedings of the 2019 15th International Conference on Distributed Computing in Sensor Systems (DCOSS), Santorini Island, Greece, 29-31 May 2019; pp. 147-156. [CrossRef]

10. Ortín, J.; Cesana, M.; Redondi, A. Augmenting LoRaWAN Performance With Listen Before Talk. IEEE Trans. Wirel. Commun. 2019, 18, 3113-3128. [CrossRef]

11. Petajajarvi, J.; Mikhaylov, K.; Pettissalo, M.; Janhunen, J.; Linatti, J. Performance of a low-power wide-area network based on LoRa technology: Doppler robustness, scalability, and coverage. Int. J. Distrib. Sens. Netw. 2017, 13, 1-16. [CrossRef]

12. Bor, M.C.; Roedig, U.; Voigt, T.; Alonso, J.M. Do LoRa low-power wide-area networks scale? In Proceedings of the 19th ACM International Conference Model. Anal. Simulat. Wireless Mobile Syst. (MSWiM), New York, NY, USA, $13-17$ November 2016.

13. LoRa ${ }^{\mathrm{TM}}$ Modulation Basics; AN1200.22, Rev. 2; Semtech Co.: Camarillo, CA, USA, 2015.

14. ITU. Itu Asp Coe Training On. In Proceedings of the Developing the ICT Ecosystem to Harness IoTs, Sami TABBANE, Bangkok, Thailand, 13-15 December 2016. 